\title{
Hemodiyaliz Fistül ve Greftlerinin Disfonksiyonlarına Girişimsel Radyoloji Yaklaşımı
}

\author{
IşıI YıIdız®, Kosti Can Çalışkan®
}

Acıbadem Üniversitesi Atakent Hastanesi, Radyoloji, İstanbul, Türkiye

Işıl Yıldız, Uzm. Dr. Kosti Can Çalışkan, Prof. Dr.
İletişim:

Uzm. Dr. |şıl YıIdız

Aclbadem Üniversitesi Atakent Hastanesi,

Radyoloji, ístanbul, Türkiye

Tel: +905323560244

E-Posta: drisilyildiz@gmail.com
Gönderilme Tarihi : 17 Kasım 2017

Revizyon Tarihi ：05 Aralık 2017

Kabul Tarihi : : 06 Aralık 2017
ÖZET

Fistül disfonksiyonunun en sık nedeni stenozlardır. Stenozlar, akut tromboza neden olarak fistülün durmasına neden olabilir. Diyaliz hastalarının mortalite ve morbiditesi hemodiyaliz kalitesi ile direk ilişkilidir bu nedenle AV fistüllerdeki stenozların tedavisi oldukça önemlidir.

Günümüzde; stenoz ve trombozlar ; stent yerleştirilmesi veya balon dilatasyon ve bir çok pıhtı çözücü teknikleri içeren girişimsel radyoloji teknikleri ile tedavi edilebilir .

Perkutan transluminal anjioplasti ile dilatasyon farklı boyutlardaki damarlar için $3-16 \mathrm{~mm}$ çapta ve $2-16 \mathrm{~cm}$ uzunlukta, şişirilebilen balonlar içeren kataterlerle uygulanır.

Tromboze fistüllerin rekanalizasyonu için farmakolojik veya mekanik yöntemler veya her iki yöntem birlikte kullanılabilir. Farmakolojik yöntemlerde trombolitiklerin lokal infüzyonu kullanılır.

Greftlerde konvansiyonel cerrahinin endovasküler yaklaşımdan biraz daha etkili ve kalııı olduğu gösterilmiştir.

Immatur fistüllerde ise minimal invaziv olduğu ve venöz rezerv korunduğu için genellikle endovasküler girişim tercih edilmektedir.

Cerrahi ve girişimsel radyoloji işbirliği ve doğru zamanda, doğru şekilde müdahale edilmesi AV yolla hemodiyalize giren hastaların yaşam sürelerinin uzatılmasında son derece önemlidir.

Anahtar sözcükler: Perkutan transluminal anjyoplasti, stent, trombektomi, vasküler girişim, tromboz

\section{INTERVENTIONAL RADIOLOGY APPROACH TO HEMODIALYSIS FISTULA AND GRAFT DYSFUNCTION}

\section{ABSTRACT}

Stenosis is the most frequent problem causing an access dysfunction. We know that tight stenosis is the underlying reason for acute thrombosis in vast majority of the cases. Since the morbidity and mortality of these patients are directly related to the effectiveness of hemodialysis, treatment of the stenosis is of great importance.

Dilatation with percutaneous transluminal angioplasty is performed with catheters containing inflatable balloons of 3-16 mm in diameter and 2-16 cm in length for vessels of different sizes.

Pharmacological methods or mechanical methods or both can be used for the recanalization of thrombosed fistulas. Pharmacological methods use local infusion of thrombolytics.

Conventional graft surgery has been shown to be more effective and lasting more than the endovascular approach. Immature fistulas are minimally invasive and endovascular intervention is generally preferred because the venous reserve is preserved.

Surgery and interventional radiology cooperation and correct intervention at the right time are crucial in prolonging the life span of hemodialysis patients.

Keywords: Percutaneous transluminal angioplasty, stent, thrombectomy, vascular intervention, thrombosis 
on dönem böbrek hastası sayısı her geçen gün artmaktadır.Bu hastalarda en çok tercih edilen hemodiyaliz giriş yolu düşük enfeksiyon oranları ve uzun süreli kullanıma imkan vermesi nedeniyle de novo arteriovenöz (AV) fistüllerdir. Fistül disfonksiyonunun en sık nedeni stenozlardır. Stenozlar, akut tromboza neden olarak fistülün kapanmasına neden olabilir. Diyaliz hastalarının mortalite ve morbiditesi hemodiyaliz kalitesi ile direk ilişkilidir bu nedenle AV fistüllerdeki stenozların tedavisi oldukça önemlidir.

Nativ fistül ve greft trombozlarının perkutan tedavisi ile ilgili tüm yayınlarda, olguların büyük çoğunluğunda altta yatan ciddi stenoz olduğu fakat stenozun maskelendiği belirtilmiştir. Bu da şu anlama gelmektedir, stenozlar farkedilip tedavi edilirse trombozların önüne geçilebilir. Günümüzde; stenoz ve trombozlar; stent yerleştirilmesi veya balon dilatasyon ve pıhtı çözme teknikleri gibi girişimsel yöntemlerle tedavi edilebilir (9).

\section{Stenozlara yönelik balon dillatasyon ve stent yerleştirilmesi}

Perkutan transluminal anjioplasti ile dilatasyon farklı boyutlardaki damarlar için $3-16 \mathrm{~mm}$ çapta ve $2-16 \mathrm{~cm}$ uzunlukta, şişirilebilen balonlar içeren kataterlerle uygulanır.

Sert, fibrotik venöz stenozların büyük çoğunluğunun dilate edilebilmesi için genellikle 20atm basınca kadar şişirilebilen yüksek basınçlı özel balonlar gereklidir. Bunların yetersiz olduğu durumlarda kesici balonlar kullanılabilir.

Stentler seçilmiş vakalarda kullanılmalıdır. Çünkü stenozlar ya stent içerisinde ya da uzantılarında tekrar edecektir. Stent içerisinde artan neointimal hiperplazi, uzun vadede rekanalizasyonu veya dilatasyonu olanaksızlaştırır. Stent yerleştirilmesine karar verildiyse önce ileride hemodializ için kullanılabilecek giriş bölgeleri belirlenmelidir. Örneğin; subklavyan vene yerleştirilen bir stent patent internal juguler ven girişine uzanmamalıdır çünkü bu ven ileride santral kateter yerleştirmek için kullanılabilir. Juguler ven kateter için en çok tercih edilen yoldur (2). Benzer şekilde, brakiyosefalik vene stent konulması planlanıyor ise stent vena cava superiora uzatılmamalıdır. Vena cava superiora uzanan stent kontralateral brakiosefalik vende ekstrinsik stenoza neden olabilir. Ekstrinsik stenoz da bu damara kateter yerleştirilmesi durumunda aynı taraf üst ekstremitede ödeme neden olabilir.

Balona bağlı gelişen akut rüptürlerde ilk yapılması gereken balonu düşük basınçla, uzun süreli rüptür olan bölgede bekletmektir. Bu başarısız olursa stentleme düşünülmelidir
(3). Ancak stent yerleştirmenin ana endikasyonu elastik rekoildir: bazı vakalarda stenoz, balon şişirildiğinde tamamen ortadan kalkar fakat balon indirildiğinde venöz duvar yeniden eski halini alır ve stenoz oluşur. Bu olay santral venlerde sık görülür, bu yüzden bazı çalışma grupları, bu bölgelere primer stent yerleştirmenin gerekli olduğunu düşünürler.

\section{Tromboze fistüllerin rekanalizasyonu}

Bu işlem için farmakolojik veya mekanik yöntemler veya her iki yöntem birlikte kullanılabilir. Farmakolojik yöntemlerde trombolitiklerin lokal infüzyonu kullanılır (ürokinaz, rTPA). Mekanik tromboaspirasyon, kateterle manuel olarak (4), çok delikli kateterlerden yüksek basınçlı cihazlar ile SIVı püskürtülmesi (5), pıhtıların Fogarty tipi kataterlerle alınması (6) ile Venturi etkisi ile vorteks oluşturan cihazlar ile trombektomi (7) ve damar duvarı üzerine direkt mekanik etkisi olan cihazların kullanımı (8) ile yapılabilir.

\section{Stenoz ve /veya trombozlara yaklaşım: Endovasküler mi cerrahi mi ?}

Her ne kadar santral ven stenozlarının vasküler tedavisinde genel bir uzlaşı olsa da, periferal stenoz veya daha sonra oluşan pıhtılaşmanın konvansiyonel cerrahi ile mi yoksa başta tanımladığımız gibi girişimsel radyologlar tarafından uygulanan endovasküler teknikler ile mi tedavi edileceği konusunda uzlaşı yoktur.

Nefrologlar tedavi için 5 şartın gerekli olduğunu belirtmektedirler: minimal invaziv, güvenlik, etkinlik, süregenlik ve venöz rezervlerin korunması. Literatür incelendiğinde ne cerrahinin, ne de girişimsel radyoloji uygulamalarının tüm bu koşulları karşılamadığı görülmektedir. Buna ek olarak, hastanın venöz rezervleri yaşam süresini belirlemektedir. Özellikle gençlerde venöz rezervlerin korunması önemlidir.

\section{Greft problemlerine yaklaşım}

Greftlerde konvansiyonel cerrahinin endovasküler yaklaşımdan biraz daha etkili ve kalıcı olduğu gösterilmiştir. Cerrahinin dezavantajı normal venlere de müdahele edildiği için venöz rezervin azalmasına neden olmasıdır (10).

Bununla beraber greftler için konvansiyonel cerrahinin, endovasküler yöntemlerden üstün olduğunu gösteren kanıt mevcut değildir.

\section{De novo AV fistüllerin izole stenozlarına yaklaşım}

Hemodiyaliz erişim yollarının büyük kısmını de nova AV fistüller oluşturmaktadır. Distal önkol radiosefalik fistüllerinde izole stenozların cerrahi revizyonunun endovasküler 
tedaviden daha başarılı olduğu düşünülmektedir ancak cerrahi tedavi ile ilgili yayın sayısı azdır $(1,11)$.

Finlandiya da yapılan bir çalışmada tüm stenozlara uygulanan endovasküler dilatasyonlar içinde, el bileği düzeyindeki anastomoz stenozlarının endovasküler dilatasyonunun en başarısız grup olduğunu gösterilmiştir (12). Bu gibi stenozların cerrahi olarak yeni anastomozlar oluşturarak tedavi edilmesi gerektiği ile ilgili yeterli bilgi vardır. Bununla beraber, randomize çok merkezli çalışmalar bu lokalizasyonlardaki stenozlara yönelik cerrahi girişimin daha üstün olduğuna dair kanıta sahip değildir.

\section{Stenozun neden olduğu gecikmiş maturasyon}

Herhangi bir yerleşimdeki de novo fistüllerin gelişen stenozlarında girişimsel radyolojinin cerrahiden daha etkin veya kalıcı olduğunu gösteren randomize bir çalışma bulunmamaktadır $(1,13)$. Minimal invaziv olması ve venöz rezervi koruması nedeniyle genellikle endovasküler girişim tercih edilmektedir.

Buna ek olarak, immatür fistüller ve akut tromboze fistüllerde, trombozun nedeni olan stenoz ön kolun distal kesiminde yerleşmediği sürece, girişimsel radyolojinin cerrahiden daha etkili olduğu düşünülmektedir.

Arter veya vendeki uzun segment/multipl darlıklar nedeniyle olgunlaşmamış fistüller balon dilatasyon ile tedavi

\section{Kaynaklar}

1. Turmel-Rodrigues L, Pengloan J, Bourquelot P. Interventional radiology in hemodialysis fistulae and grafts: a multidisciplinary approach. Cardiovasc Intervent Radiol 2002; 25: 3-16. [CrossRef]

2. Turmel-Rodrigues $L$, Bourquelot $P$, Raynaud A, Sapoval M. Primary stent placement in hemodialysis-related central venous stenoses: the dangers of a potential 'radiologic dictatorship'. Radiology 2000;217: 600-2. [CrossRef]

3. Raynaud AC, Angel CY, Sapoval MR, Beyssen B, Pagny JY, Auguste M. Treatment of hemodialysis access rupture during PTA with Wallstent implantation. J Vasc Interv Radiol 1998; 9: 437-42.

4. Turmel-Rodrigues L, Sapoval M, Pengloan J et al. Manual thromboaspiration and dilation of thrombosed dialysis access: midterm results of a simple concept. J Vasc Interv Radiol 1997; 8: 813-24.

5. Valji K, Bookstein JJ, Roberts AC, Oglevie SB, Pittman C, O'Neil MP. Pulse-spray pharmacomechanical thrombolysis of thrombosed hemodialysis access grafts: long-term experience and comparison of the original and current techniques. Am J Radiol 1995;164:1495500. [CrossRef]

6. Trerotola SO, Lund GB, Scheel PJ, Savader SJ, Venbrux AC, Osterman FA. Thrombosed hemodialysis access grafts: percuta- neous mechanical declotting without urokinase. Radiology 1994; 191: 721-6. [CrossRef] edilebilir. Bu vakalarda bir yıllık primer patensi \%80, sekonder patensi $\% 40$ olarak rapor edilmiştir (16). Tromboze fistüllerde endovasküler tedavi başarı oranları \%76 ve \%94 olarak bildirilmiştir $(1,14)$. Cerrahi tedavi bu durumda başarısız olduğundan Amerikalı cerrahlar tromboze otolog fistüllere cerrahi olarak müdahale etmediklerini bildirmişlerdir $(15,16)$.

\section{Tecrübe ve görüntülemenin önemi}

$\mathrm{Bu}$ işle ilgilenen girişimsel radyolog sayısının artması ile endovasküler tedavi başarısı artmıştır. Fistül görüntülemesi optimal yapılmadan başarılı tedavi yapılması mümkün değildir. İyi görüntüleme ise gelişmiş anjiyografi ünitelerinde yapılabilir. Eğer fistülünde problem olan hastalar bu işle ilgilenen bir girişimsel radyologa yönlendirilirse, tek seansta optimal görüntüleme ve tedavi yapılabilir.

\section{Sonuç}

El bilek fistüllerinde anostomoz bölgesindeki izole stenozlar haricinde AV fistüllerin stenoz ve trombozlarında tedavi için ilk seçenek perkutan endovasküler tedavi olmalıdır. Bu sayede venöz rezerv daha uzun süre korunabilmektedir. Cerrahi ancak hızlı tekrarlayan stenozlarda gündeme gelmelidir. Cerrahi ve girişimsel radyoloji işbirliği ve doğru zamanda, doğru şekilde müdahale edilmesi AV yolla hemodiyalize giren hastaların yaşam sürelerinin uzatılmasında son derece önemlidir.

7. Sofocleous CT, Cooper SG, Schur I, Patel RI, Iqbal A, Walker S. Retrospective comparison of the Amplatz-Thrombectomy- Device with modified pulse-spray pharmacomechanical thrombolysis of the thrombosed hemodialysis access grafts. Radiology 1999;213:561-7. [CrossRef]

8. Trerotola SO, Vesely TM, Lund GB, Soulen MC, Ehrman KO, Cardella JF. Treatment of thrombosed hemodialysis access grafts: ArrowTrerotola percutaneous thrombolytic device versus pulse-spray thrombolysis. Radiology 1998; 206: 403-14. [CrossRef]

9. Green LD, Lee DS, Kucey DS. A metaanalysis comparing surgical thrombectomy, mechanical thrombectomy, and pharmacomechanical thrombolysis for thrombosed dialysis grafts. J Vasc Surg 2002; 36: 939-45.

10. Oakes DD, Sherck JP, Cobb LF. Surgical salvage of failed radiocephalic arteriovenous fistulae: techniques and results in 29 patients. Kidney Int 1998; 53: 480-7. [CrossRef]

11. Manninen HI, Kaukanen ET, Ikäheimo R, Karhapää P, Lahtinen T, Matsi $P$, et al. Brachial arterial access: endovascular treatment of failing Brescia-Cimino hemodialysis fistulas--initial success and long-term results. Radiology 2001;218:711-8. [CrossRef]

12. Turmel-Rodrigues L, Pengloan J, Baudin S, Testou D, Abaza M, Dahdah G, et al. Treatment of stenosis and thrombosis in haemodialysis fistulas and grafts by interventional radiology. Nephrol Dial Transplant 2000; 15: 2029-36. [CrossRef] 
13. Turmel-Rodrigues L, Pengloan J, Rodrigue $H$, Brillet $G$, Lataste $A$, Pierre $D$, et al. Treatment of failed native arteriovenous fistulae for hemodialysis by interventional radiology. Kidney Int 2000; 57: 112440. [CrossRef]

14. Turmel-Rodrigues $\mathrm{L}$, Mouton A, Birmelé B, Billaux L, Ammar N, Grézard O, Hauss S, Pengloan J, et al. Salvage of immature forearm fistulas for haemodialysis by interventional radiology. Nephrol Dial Transplant 2001; 16: 2365-71. [CrossRef]
15. Hingorani A, Ascher E, Kallakuri S, Greenberg S, Khanimov Y. Impact of reintervention for failing upper-extremity arteriovenous autogenous access for hemodialysis. J Vasc Surg 2001; 34: 1004-9. [CrossRef]

16. Berman SS, Gentile AT. Impact of secondary procedures in autogenous arteriovenous fistula maturation and maintenance. J Vasc Surg 2001;34: 866-71. [CrossRef] 